\title{
Impact and mechanisms of cigarillo flavor descriptors on susceptibility to use among young adult nonusers of tobacco
}

\author{
Aya Avishai $^{1,2}$ (D) | Clare Meernik ${ }^{3}$ (D) | Adam O. Goldstein ${ }^{2,4}$ | Allison J. Lazard ${ }^{2,5}$ (D) | \\ Leah M. Ranney ${ }^{4}$ Paschal Sheeran ${ }^{1,2}$
}

${ }^{1}$ Department of Psychology and Neuroscience, University of North Carolina at Chapel Hill, Chapel Hill, NC, USA

${ }^{2}$ Lineberger Comprehensive Cancer Center, University of North Carolina at Chapel Hill, Chapel Hill, NC, USA

${ }^{3}$ Department of Epidemiology, University of North Carolina at Chapel Hill, Chapel Hill, NC, USA

${ }^{4}$ Department of Family Medicine, University of North Carolina at Chapel Hill, Chapel Hill, NC, USA

${ }^{5}$ Hussman School of Journalism and Media, University of North Carolina at Chapel Hill, Chapel Hill, NC, USA

\section{Correspondence}

Aya Avishai, Department of Psychology and Neuroscience, University of North Carolina at Chapel Hill, Davie Hall, Chapel Hill, NC 27599, USA.

Email: avishaiy@live.unc.edu

Funding information

National Cancer Institute, Grant/Award Number: P50CA180907; Lineberger

Comprehensive Cancer Center, University of North Carolina, Grant/Award Number:

Developmental Award

\begin{abstract}
Evidence suggests that flavoring may impact reactions to tobacco products. In the present research, we tested the impact and mechanisms of cigarillo flavor descriptors on susceptibility to use in order to determine whether exposure to cigarillos with characterizing flavors increases susceptibility in young adult nonusers of tobacco and, if so, why susceptibility increases. Nonsmoking, 18-26 years old U.S. residents were recruited for an online randomized controlled trial with two conditions: cigarillos with characterizing flavors (experimental condition) versus cigarillos with tobacco flavors (control condition). Experimental condition participants $(n=49)$ were presented with five cigarillo pack images with characterizing flavors (e.g., "Sweet"), whereas control condition participants $(n=53)$ were presented with five standard, tobacco-flavored images (e.g., "Air-Cured"). Each presented cigarillo image included a description of the pack flavor. Approach bias to the cigarillos was measured using the Implicit Association Test, and participants reported their perceptions of taste, smell, social acceptability, enjoyment, and harm in relation to each cigarillo pack. Finally, participants indicated their susceptibility to using cigarillos. Susceptibility to cigarillo use was significantly greater for participants exposed to the cigarillo packs with characterizing flavors. Taste perceptions both mediated and moderated the relationship between cigarillo flavor descriptors and susceptibility to use. Characterizing flavors increased susceptibility to cigarillo use via two routes: (1) by enhancing perceptions of taste and (2) by increasing the strength of association between perceptions of taste and susceptibility. These findings have implications for public health and policy decisions regarding banning or limiting characterizing flavors in cigarillos.
\end{abstract}

\section{1 | INTRODUCTION}

Tobacco use continues to be a substantial public health concern as approximately one in five adults in the U.S. consumes tobacco products (Wang et al., 2018). Thus, research examining the factors contributing to smoking uptake and susceptibility among nonusers helps shape public policy. In particular, research regarding flavoring in tobacco products has become salient as a greater number of flavored tobacco products have become available for purchase. The present research aims to explore (1) whether characterizing flavors in cigarillos increases smoking susceptibility in young adult nonusers of tobacco, and (2) how characterizing flavors increase susceptibility to cigarillo use.

Our focus on characterizing flavors in tobacco is partly due to their popularity-approximately $80 \%$ of adolescent, $73 \%$ of young adult, and $46 \%$ of older adult tobacco users report using flavored tobacco (Harrell, Loukas, Jackson, Marti, \& Perry, 2017; Villanti et al., 2017). Previous work has found that the majority of youth 
and young adults who are newly using tobacco first tried a tobacco product with characterizing flavor (Villanti et al., 2019). Additionally, when compared with first using a tobacco-flavored product, first using a tobacco product with characterizing flavor is positively related to later tobacco use. While tobacco with characterizing flavors is not allowed in cigarettes in the United States, there are numerous characterizing flavor options on the market for cigarillos, such as sweet, grape, tropical, wine, jazz, blueberry, strawberry, pineapple, mango, and many more. The prevalence of tobacco with characterizing flavors raises the question, why is it so popular? While past research and reviews mainly focused on attitudes toward and beliefs about flavored tobacco (Feirman, Lock, Cohen, Holtgrave, \& $\mathrm{Li}, 2015)$, few studies have explored the perceptions of particular product attributes in flavored tobacco (Huang et al., 2017).

\section{1 | Explicit perceptions of tobacco products}

Systematic reviews have indicated that nonmenthol flavored tobacco products are associated with increased appeal ratings, decreased harm ratings, and decreased quit intentions (Feirman et al., 2015; Huang et al., 2017; Kowitt et al., 2017). Focus group research also suggests that young people may feel that characterizing flavors contribute to their social lives and are part of the social norm (Camenga et al., 2018; Wagoner et al., 2016). Thus, we chose to examine perceptions of appeal, harm, and social acceptability of cigarillos in the present research. While perceptions of harm and social acceptability are relatively straightforward to measure through selfreport, there are many ways to measure a product's appeal. Based on prior research, we focused on three key dimensions of product appeal, namely, taste, smell, and enjoyment (Meernik et al., 2018). Qualitative data indicate that both the taste and smell of flavored tobacco products are important to smokers, and elicit positive emotional responses (Sterling, Fryer, Nix, \& Fagan, 2015).

\section{2 | Implicit approach bias toward tobacco products}

Prior research has shown that even after accounting for social influences, greater exposure to tobacco marketing is positively associated with ever smoking cigarettes among adolescents (Henriksen, Feighery, Wang, \& Fortmann, 2004). Studies have examined the potential mechanisms by which marketing influences smoking behavior and found that tobacco promotions increase positive attitudes toward, and beliefs about, tobacco use. In turn, positive attitudes and beliefs increase tobacco use intentions and rates of uptake (review by DiFranza et al., 2006). Another potential mechanism of marketing that has been largely overlooked in research on tobacco is unconscious (implicit) bias. One domain where unconscious bias may especially influence smoking behavior concerns tobacco products with characterizing flavors, as marketing can exploit people's rich store of associations with well-liked flavors.

Research suggests that tobacco products with characterizing flavors are viewed more favorably both by users and nonusers than tobacco-flavored products (systematic review by Feirman et al., 2015). We suggest that this may be partially explained by implicit approach bias; that is, tobacco products with appealing characterizing flavors may elicit an automatic "approach" response as compared to tobacco-flavored products. In order to capture automatic motivational tendencies, researchers typically use timed reaction tasks such as the Implicit Association Test (IAT; Greenwald, McGhee, \& Schwartz, 1998). In the approach bias IAT, participants categorize approach/avoid words (e.g., toward, away) and pictures (e.g., of tobacco products) as quickly and accurately as possible. Approach bias is indexed by the relative speed of approach versus avoidance responses to the products. Prior research indicates that the IAT is a valid index of approach bias in health domains. In a study of alcohol consumption, IAT scores were related to self-reported cue reactivity, alcohol motivation, and alcohol behavior (Ostafin \& Palfai, 2006). In the present research, we applied the IAT procedure using pictures of tobacco products with characterizing flavors and tobacco products with tobacco flavors. To our knowledge, implicit responses to flavor descriptors in tobacco have never before been tested.

\section{3 | Is susceptibility to use mediated or moderated by implicit and explicit perceptions?}

Our key prediction is that characterizing flavors will increase susceptibility to cigarillo use; our next aim is to explore the mechanisms by which this occurs. We hypothesize that there are two ways that cigarillo products with characterizing flavors could increase susceptibility to use: mediation (Figure 1, Panel A) or moderation (Figure 1, Panel B) by implicit and explicit perceptions of cigarillos. The first mechanism that we consider is mediation; that

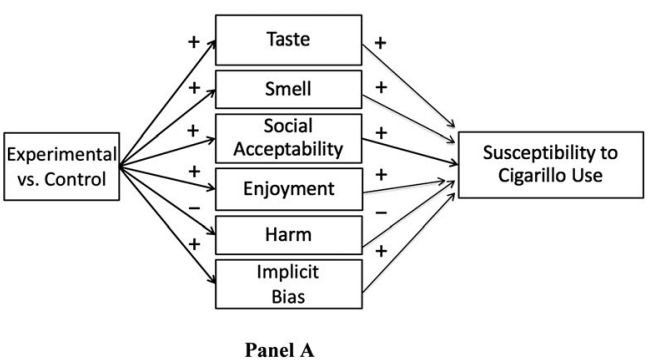

Panel A

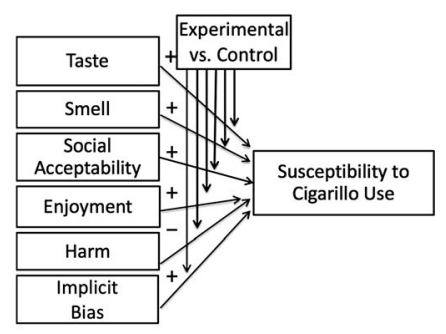

Panel B
FIGURE 1 Hypothesized mediation (Panel A) and moderation (Panel B) models 
is, that exposure to cigarillo products with characterizing flavors changes individuals' perceptions of the products, thereby influencing smoking behavior. Previous research would suggest that characterizing flavors may cause people to perceive a tobacco product more favorably-as more appealing and less harmfulthan its tobacco-flavored counterpart (Feirman et al., 2015; Huang et al., 2017; Kowitt et al., 2017). These increased appeal ratings and lowered harm ratings might then lead to increased smoking susceptibility and behavior. Under mediation, we anticipate that characterizing flavors increase appeal ratings (perceptions of taste, smell, and enjoyment) and perceived social acceptability, while decreasing perceptions of harm from the products. We anticipate that harm will be reduced both due to previous findings supporting this hypothesis (Huang et al., 2017; Kowitt et al., 2017), and also because characterizing flavors tend to decrease the harshness and biting taste of tobacco. Thus, if a tobacco product with characterizing flavors is less harsh and smoother to smoke than its tobacco-flavored counterpart, we expect that it will be perceived as less harmful to one's health. We also hypothesize that characterizing flavors increase approach bias toward the cigarillos. In this way, characterizing flavors are expected to alter implicit and explicit perceptions, thereby increasing susceptibility to cigarillo use.

The second possibility we suggest is moderation: characterizing flavors do not necessarily change individuals' perceptions of cigarillo products, but instead alter the strength of association between the perceptions of the products and cigarillo use behavior. Under moderation, we expect that characterizing flavors will not alter implicit or explicit perceptions. Instead, we anticipate that characterizing flavors will strengthen the relationships between susceptibility to use and approach bias, appeal ratings (perceptions of taste, smell, and enjoyment), and perceived social acceptability and weaken the relationship between perceived harm and susceptibility. For example, we would expect characterizing flavors to increase the association between taste perceptions and susceptibility to cigarillo use.

\section{4 | The present study}

The present study focuses on cigarillo use. The U.S. Food and Drug Administration enacted a ban on cigarettes containing certain characterizing flavors in 2009. Only recently has a ban on characterizing flavors in cigars-and therefore, cigarillos-been proposed in the United States. Much of the research regarding flavoring in tobacco products is correlational and does not permit inferences about the causal role of characterizing flavors in product perceptions and use. Thus, the present research explores how characterizing flavors influence susceptibility to smoking cigarillos using an experimental design. We directly compare perceptions of cigarillo products with characterizing flavors and those with tobacco flavors on susceptibility to use and examine the mechanisms by which characterizing flavors may lead to cigarillo use. We predict that characterizing flavors increase susceptibility to cigarillo use among young adult nonusers of tobacco. We also test the predictions that perceptions of cigarillos mediate or moderate the effect of flavor descriptors on susceptibility to use.

\section{2 | METHOD}

\section{1 | Participants}

Participants were recruited through Amazon Mechanical Turk, screened for age and tobacco use, and paid \$2.00 each for participation. They completed questionnaires and tasks using two programs, Qualtrics and Inquisit. Only individuals 18 to 26 years of age that reported that they do not smoke cigarettes, cigars, e-cigarettes, or electronic nicotine delivery systems, little cigars, or cigarillos were eligible to participate. After eligibility screening for age and tobacco use, participants were randomized to one of two conditions: tobacco-flavored cigarillos (control condition) or cigarillos with characterizing flavors (experimental condition).

The screener was taken 3,121 times, and entries were removed for the following reasons (hierarchically): Entering the survey multiple times in order to qualify ( $n=1,333)$, not meeting eligibility criteria $(n=1,617)$, incomplete Qualtrics survey data $(n=62)$, no Inquisit task data $(n=5)$, and incomplete Inquisit task data $(n=2)$. After removing these entries, 102 participants were left in the sample (53 in the control condition and 49 in the experimental condition). These were individuals who took the screener once, qualified, and completed the entire study. The sample was composed of 65 females (63.7\%), 35 males (34.3\%), and 2 nonbinary individuals (2\%), and the mean age was 23.34 years $(S D=1.99)$. There were no differences between conditions in age or gender, suggesting that randomization was successful.

\section{2 | Procedure}

Participants viewed five cigarillo pack images in various colors (pink, red, purple, green/blue, and yellow) with labeled flavors and descriptions, depending on assigned condition. After seeing each image, participants completed attention check items about the cigarillo descriptions. They then completed a measure of approach bias toward cigarillos, followed by items regarding their perceptions of taste, smell, social acceptability, enjoyment, harm, and their susceptibility to the cigarillos. Finally, participants completed a recall measure of the cigarillo flavors they saw during the study.

\section{3 | Stimuli}

All participants were informed that they would see a series of images of cigarillo flavors and that they must view the images and read the accompanying text, as they would be asked questions about the images later (see Figure 2 for sample images). In order to prevent 

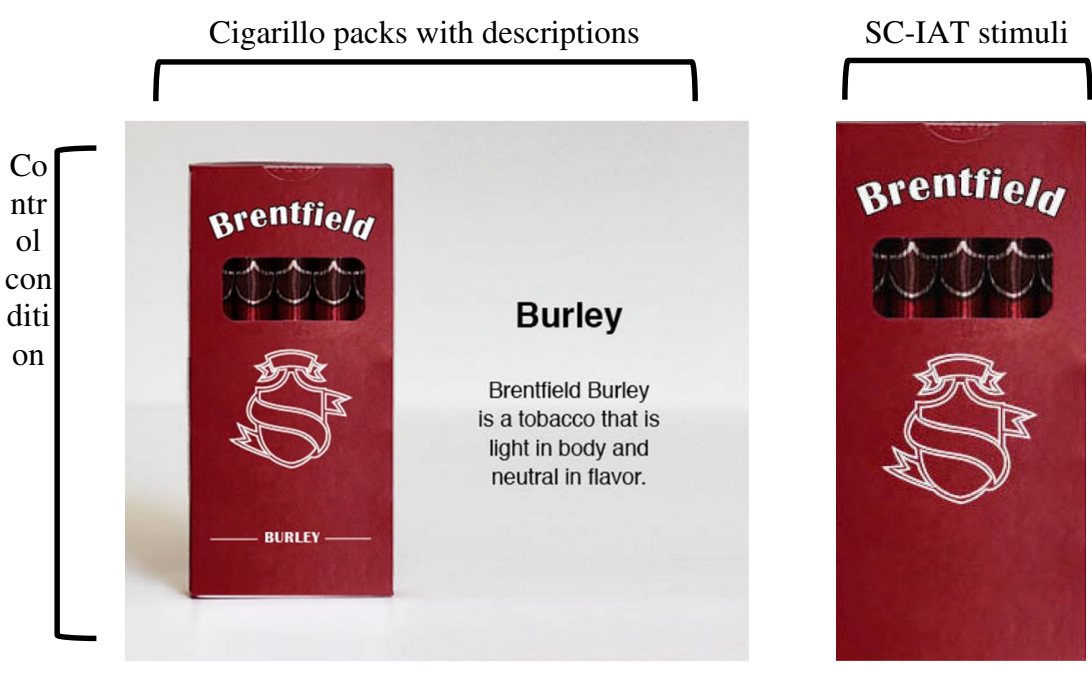

FIGURE 2 Examples of cigarillo packs presented to participants [Color figure can be viewed at wileyonlinelibrary.com]
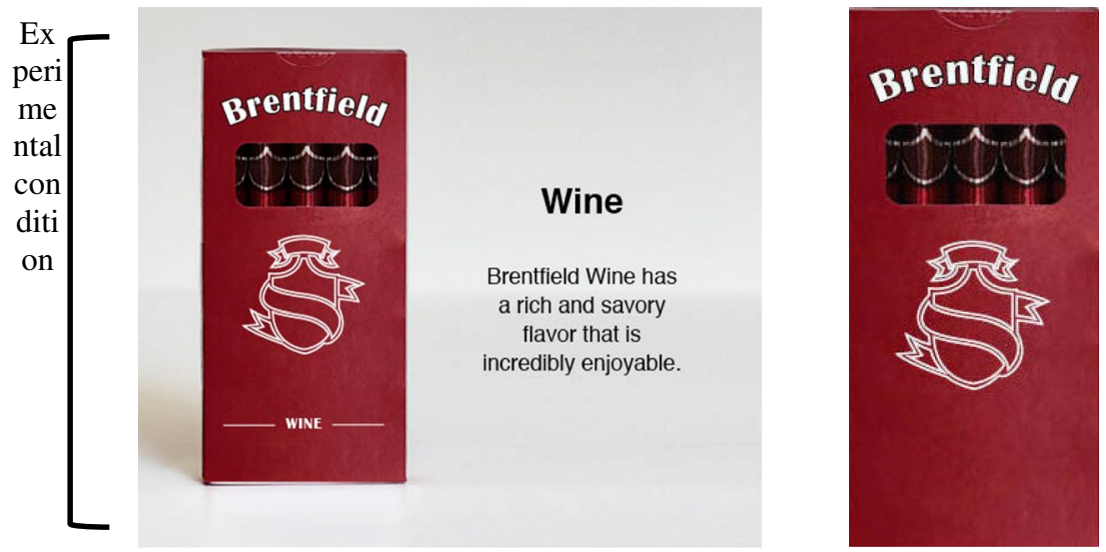

preexisting biases toward the cigarillo products, we used a fictitious brand, called "Brentfield," which has been used in previous research (Byron et al., 2018; Lazard et al., 2019; Meernik et al., 2018). Images of all stimuli used in this study can be viewed in the Online Supplementary Materials file.

\subsection{1 | Experimental condition stimuli}

Participants in the experimental condition viewed cigarillo pack images with the following characterizing flavors: (1) "Sweet" cigarillos in a pink pack, with the description "Brentfield Sweet is divinely sugary and fragrant. Its delightful flavor is a joy"; (2) "Wine" cigarillos in a red pack, with the description "Brentfield Wine has a rich and savory flavor that is incredibly enjoyable"; (3) "Grape" cigarillos in a purple pack, with the description "Brentfield Grape has a juicy and succulent fruit flavor"; (4) "Jazz" cigarillos in a green/blue pack, with the description "Brentfield Jazz has a smooth and sweet-tasting flavor"; and (5) "Tropical" cigarillos in a yellow pack, with the description "Brentfield Tropical has a sweet smell and exotic flavor. It tastes delicious and smooth." These characterizing flavors were chosen by examining the highest volume flavored cigar sales (Swedish Match North America, 2015); the order of their presentation in the study was randomized.

\subsection{2 | Control condition stimuli}

Participants in the control condition viewed cigarillo pack images with the following tobacco flavors: (1) "Dark-Fired" cigarillos in a pink pack, with the description "Brentfield Dark-Fired tastes strong and smoky. Its woody nuances also add to its earthy notes"; (2) "Burley" cigarillos in a red pack, with the description "Brentfield Burley is a tobacco that is light in body and neutral in flavor"; (3) "Flue-Cured" cigarillos in a purple pack, with the description "Brentfield Flue-Cured is heat-dried and has a powerful, savory flavor"; (4) "Air-Cured" cigarillos in a green/blue pack, with the description "Brentfield Air-Cured gives the smoke a light flavor"; and (5) "Rustica" cigarillos in a yellow pack, with the description "Brentfield Rustica is stalk-cut and sun-cured in fields. It has a concentrated smell and flavor." As in the experimental condition, the order of the flavors being presented was randomized.

\section{4 | Measures}

\subsection{1 | Implicit perception: Approach bias}

Approach bias to cigarillos was measured (using the Inquisit program) through an approach/avoidance Single Category Implicit Association Test (SC-IAT; Karpinski \& Steinman, 2006) modeled after Ostafin and 
Palfai's (2006) IAT. The attribute stimuli used were approach words (approach, forward, advance, closer, and toward) and avoidance words (avoid, escape, withdraw, away, and leave) and the target stimuli were pictures of cigarillo packs. The packs were colored as before but had no labels (aside from the Brentfield logo and symbol) and no text descriptions (e.g., see Figure 2). During the SC-IAT, there was a compatible practice block (24 trials), a compatible test block (72 trials), an incompatible practice block (24 trials), and an incompatible test block (72 trials). Compatible blocks paired Brentfield with approach, while incompatible blocks paired Brentfield with avoidance. The order of the blocks (compatible first, or incompatible first) was counterbalanced between participants. Each trial had a pretrial pause of 250 milliseconds, and stimuli were presented until a correct response was given. In this way, incorrect responses had built-in error penalties that extended the latencies for those trials. We did not display a reminder to participants to respond more quickly during the task if latencies exceeded a certain limit. Only test blocks were used to calculate the D-values. Mean latencies of responses were computed for each test block (i.e., separately for the compatible and incompatible test blocks) by dividing the sum of latencies by the number of trials for each test block. An overall standard deviation of latencies was also computed for each participant, across both blocks. A D-value was calculated for each participant by subtracting the mean latency for the compatible block from the mean latency for the incompatible block, and then, dividing the resulting difference by the overall standard deviation. Thus, greater D-values indicated that participants associated "Brentfield" more with "approach" than "avoid," and therefore, had more approach bias to cigarillos. The approach/avoid SC-IAT was modeled on an established test by Palfai and Ostafin (2003); we present an image of the SC-IAT in the Online Supplementary Materials file.

\subsection{2 | Explicit perceptions}

Appeal ratings (taste, smell, and enjoyment), social acceptability ratings, and harm ratings were measured using self-reports. Participants were asked about their perceptions of the five cigarillo flavors to which they were exposed, first on taste ("Overall, how much would you like the taste of Brentfield [flavor]?" 0 = Not at all, $100=$ Very much, $\alpha=0.90$ ) and smell ("Overall, how much would you like the smell of Brentfield [flavor]?" $0=$ Not at all, $100=$ Very much, $\alpha=0.90$ ). They then responded to the item "I would expect that smoking Brentfield [flavor] would be..." in regards to "socially acceptable" ( $\alpha=0.97)$, "enjoyable" ( $\alpha=0.93)$, "pleasant" ( $\alpha=0.93)$, "harmful" ( $\alpha=$ 0.95), and "safe" ( $\alpha=0.96), 0=$ Not at all, $100=$ Very much (modified from Wakefield et al., 2012, and from Van De Ven, Engels, Otten, \& Van Den Eijnden, 2007). The "enjoyable" and "pleasant" items were averaged to form a measure of enjoyment $(r=0.95, p<.001)$, and the "harmful" and "safe" items were averaged to form a measure of harm after the "safe" item was reverse-scored $(r=0.59, p<.001)$. The order of flavors and items was randomized.

\subsection{3 | Susceptibility to cigarillo use}

Finally, participants rated "How much does seeing this pack make you want to try Brentfield [flavor]?" (0 = Not at all, 100 = Very much; $\alpha=$ 0.91; modified from Pepper, Emery, Ribisl, Southwell, \& Brewer, 2014) and "If one of your friends were to offer you a Brentfield [flavor], would you smoke it?" (0 = Definitely no, 100 = Definitely yes; $\alpha=0.94$; modified from Gritz et al., 2003 and from Pierce, Choi, Gilpin, Farkas, $\&$ Merritt, 1996). The two items were averaged to form a measure of susceptibility $(\alpha=0.95 ; r=0.80, p<.001)$.

\section{3 | RESULTS}

\section{1 | Impact of condition on susceptibility to cigarillo use and perceptions of cigarillos}

Using a MANOVA (see Table 1), Pillai's Trace indicated that condition was a significant predictor of outcomes (Intercept: $F(7,94)=$ 591.459, $p<.001, \eta_{p}{ }^{2}=0.978$; Condition: $F(7,94)=2.553, p=.019$, $\left.\eta_{p}{ }^{2}=0.160\right)$. We found that participants in the experimental condition had increased susceptibility to cigarillos ( $F=10.85, p=.001)$. The experimental condition also led to more favorable perceptions of cigarillo taste $(F=10.68, p=.001)$ and smell $(F=13.85$, $p<.001$ ), and increased perception of cigarillos as socially acceptable
TABLE 1 Effect of condition on implicit and explicit perceptions of cigarillos and susceptibility to use using MANOVA

\begin{tabular}{|c|c|c|c|c|c|}
\hline \multirow[b]{2}{*}{ Outcome } & \multicolumn{5}{|l|}{ Condition } \\
\hline & $\begin{array}{l}\text { Control } \\
(N=53)\end{array}$ & $\begin{array}{l}\text { Experimental } \\
(N=49)\end{array}$ & $F$ & $p$ & $\eta_{p}{ }^{2}$ \\
\hline Approach bias & $0.02(0.23)$ & $0.01(0.25)$ & 0.08 & .781 & 0.001 \\
\hline Taste & $20.83(19.96)$ & $35.18(24.33)$ & 10.68 & .001 & 0.096 \\
\hline Smell & $23.70(21.88)$ & $41.47(26.28)$ & 13.85 & $<.001$ & 0.122 \\
\hline Social acceptability & $30.11(25.54)$ & $44.00(26.51)$ & 7.26 & .008 & 0.068 \\
\hline Enjoyment & $22.59(21.43)$ & $35.90(24.15)$ & 8.70 & .004 & 0.080 \\
\hline Harm & $82.38(19.58)$ & 78.41 (19.29) & 1.06 & .305 & 0.011 \\
\hline Susceptibility & $14.42(16.90)$ & 27.57 (23.18) & 10.85 & .001 & 0.098 \\
\hline
\end{tabular}

Note: Standard deviations are in parentheses. 
$(F=7.26, p=.008)$ and enjoyable $(F=8.70, p=.004)$. However, condition did not significantly predict approach bias $(F=0.08, p=.78$ ) or perceptions of harm $(F=1.06, p=.31)$.

We note that the MANOVA in Table 1 was adequately powered to test our hypotheses. G*Power estimated our observed (post hoc) power for condition as .895 (based on an effect size $\mathrm{f}^{2}(\mathrm{~V})$ of .1904762 computed from a Pillai $\vee$ of .160 , alpha level of .05, total sample size of 102, 2 groups, and 7 response variables), while SPSS estimates our observed power as .864 .

\section{2 | Perceptions of cigarillos predicting susceptibility to use}

Perceptions of taste $(r=0.81, p<.001)$, smell $(r=0.74, p<.001)$, social acceptability $(r=0.47, p<.001)$, enjoyment $(r=0.80, p<.001)$, and harm $(r=-0.58, p<.001)$ each predicted susceptibility to cigarillo use. Approach bias did not significantly predict susceptibility $(r=0.05, p=.61)$. Next, we conducted a simultaneous regression with the implicit and explicit perception variables predicting susceptibility. In the simultaneous regression, only taste $(\beta=0.39, p<.05)$ and harm $(\beta=-0.20, p<.01)$ significantly predicted susceptibility (Table 2). Higher taste ratings predicted greater susceptibility, whereas higher harm ratings predicted reduced susceptibility.

TABLE 2 Regression of susceptibility to use on implicit and explicit perceptions of cigarillos

\begin{tabular}{|llll|} 
& B & SE & $\beta$ \\
\hline Approach bias & -1.40 & 1.27 & -0.066 \\
\hline Taste & 8.20 & 3.72 & $0.388^{*}$ \\
\hline Smell & 3.13 & 2.35 & 0.148 \\
\hline Social acceptability & -1.95 & 1.67 & -0.092 \\
\hline Enjoyment & 5.70 & 3.49 & 0.270 \\
\hline Harm & -4.26 & 1.42 & $-0.202^{* *}$ \\
\hline Model F & & & $38.64^{* * *}$ \\
\hline$R^{2}$ & & & $0.71^{* * *}$ \\
\hline
\end{tabular}

${ }^{*} p<.05 ;{ }^{* *} p<.01 ;{ }^{* * *} p<.001$.

\section{3 | Perceptions as mediators of impact of condition on susceptibility to cigarillo use}

The findings in Tables 1 and 2 indicate that the only variable that both significantly differed between conditions and significantly predicted susceptibility was taste perception. Additionally, when we analyzed a mediation model with all perception variables included (see Figure 3), we found that taste was the only variable that had both a significant $a$ path $(B=0.62, p<.01)$ and a significant $b$ path $(B=0.38$, $p<.05$ ). That is, when testing approach bias, appeal ratings (taste, smell, and enjoyment), social acceptability, and harm simultaneously as mediators, taste was the only variable for which both paths were significant. We confirmed this finding in a separate analysis by testing taste perception as a sole mediator of the relationship between condition and susceptibility to cigarillo use (see Figure 4). We found that taste fully mediated the relationship (c path: $\mathrm{B}=0.62, p<.01 ; \mathrm{c}^{\prime}$ path: $\mathrm{B}=0.14, p=.27$ ).

\subsection{Did condition influence how well perceptions of cigarillos predict susceptibility to use?}

Next, we ran a simultaneous linear regression with all of the implicit and explicit perception variables predicting susceptibility, separately for each condition (see Table 3 ). Ratings of taste significantly and positively predicted susceptibility in the experimental condition ( $\beta=0.69, p<.01$ ), but those ratings did not predict susceptibility in the control condition $(\beta=-0.09, p=.74)$. This further confirmed our findings regarding the unique importance of taste perceptions in

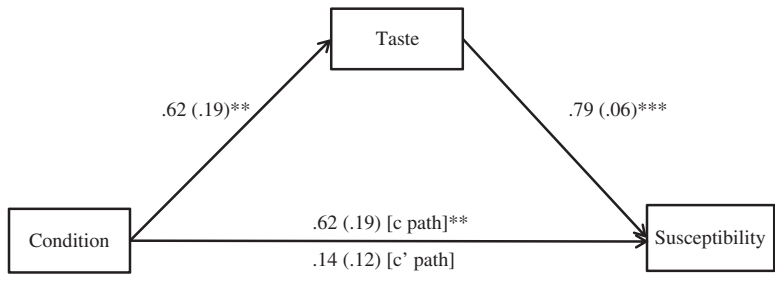

FIGURE 4 Mediation of effect of condition on susceptibility to cigarillo use by taste perceptions. For condition, control was coded as 0 and experimental as $1 .{ }^{*} p<.05,{ }^{* *} p<.01,{ }^{* * *} p<.001$

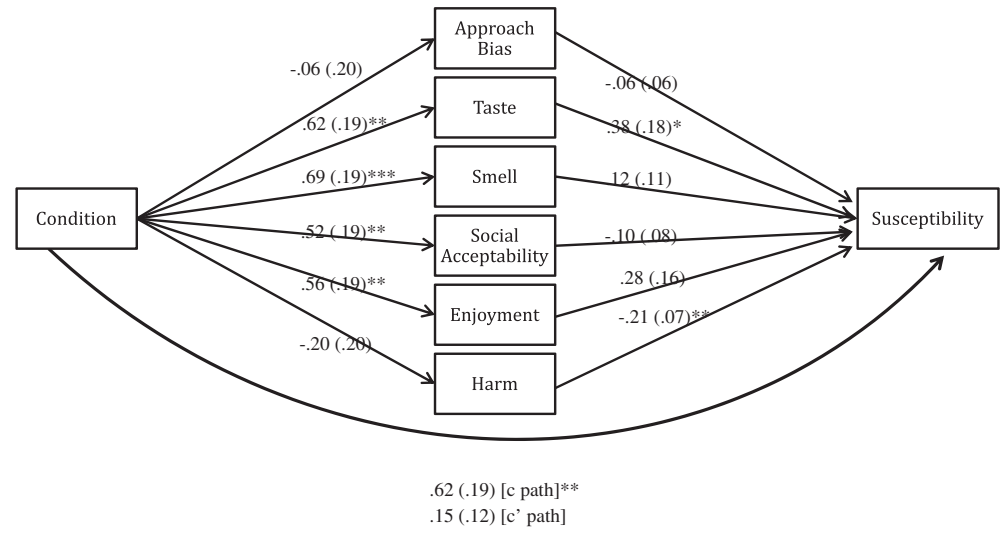

FIGURE 3 Multiple mediation of effect of condition on susceptibility to use by implicit and explicit perceptions of cigarillos. For condition, control was coded as 0 and experimental as 1 . ${ }^{*} p<.05,{ }^{* *} p<.01,{ }^{* * *} p<.001$ 
TAB LE 3 Regression of susceptibility to use on implicit and explicit perceptions of cigarillos for each condition

\begin{tabular}{|c|c|c|c|c|c|c|c|c|c|}
\hline \multirow[b]{2}{*}{ Predictor } & \multicolumn{4}{|l|}{ Control } & \multicolumn{4}{|c|}{ Experimental } & \multirow{2}{*}{$\begin{array}{l}\text { Difference } \\
\text { in B } \\
z\end{array}$} \\
\hline & $r$ & B & SE & $\beta$ & $r$ & B & $S E$ & $\beta$ & \\
\hline Approach bias & 0.10 & -0.73 & 1.68 & -0.043 & 0.04 & -1.92 & 1.96 & -0.083 & 0.458 \\
\hline Taste & $0.69^{* * *}$ & -1.51 & 4.58 & -0.089 & $0.86^{* * *}$ & 15.94 & 5.68 & $0.688^{* *}$ & $2.389^{*}$ \\
\hline Smell & $0.68^{* * *}$ & 2.34 & 2.98 & 0.139 & $0.74^{* * *}$ & 2.12 & 3.36 & 0.091 & 0.051 \\
\hline Enjoyment & $0.77^{* *}$ & 11.10 & 4.83 & $0.657^{*}$ & $0.79^{* * *}$ & 2.65 & 4.83 & 0.114 & 1.237 \\
\hline Harm & $-0.57^{* * *}$ & -3.52 & 1.94 & -0.209 & $-0.60^{* * *}$ & -3.20 & 2.26 & -1.417 & 0.108 \\
\hline Model F & & $13.67^{* * *}$ & & & & $21.98^{* * *}$ & & & \\
\hline$R^{2}$ & & $0.64^{* * *}$ & & & & $0.76^{* * *}$ & & & \\
\hline
\end{tabular}

${ }^{*} p<.05 ;{ }^{* *} p<.0 ;{ }^{* * *} p<0.001$.

TABLE 4 Interaction between taste perceptions and condition predicting susceptibility to cigarillo use

\begin{tabular}{lcll} 
& B & SE & $\beta$ \\
\hline Taste & 13.57 & 1.98 & $0.643^{* * *}$ \\
\hline Condition & 3.05 & 2.56 & 0.073 \\
\hline Taste $\times$ condition & 5.32 & 2.60 & $0.187^{*}$ \\
\hline Model F & $67.28^{* * *}$ & & \\
$R^{2}$ & $0.67^{* * *}$ & & \\
\hline
\end{tabular}

Note: For condition, control was coded as 0 and experimental as 1. For the control condition, $\mathrm{B}=13.568, t=6.8664, p<.001$. For the experimental condition, $\mathrm{B}=18.886, t=11.1985, p<.001$. ${ }^{*} p<.05 ;{ }^{* * *} p<.001$.

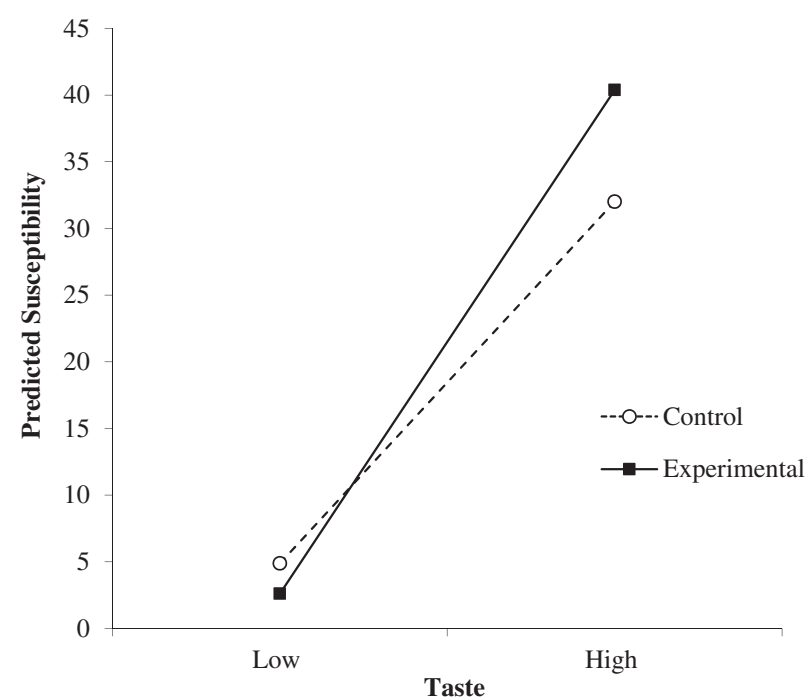

FIGURE 5 Interaction between taste perception and condition predicting susceptibility to cigarillo use

facilitating greater susceptibility among participants exposed to the cigarillos with characterizing flavors. We formally tested moderation (see Table 4 and Figure 5) by running a regression modeling the interaction between taste and condition. We found that condition significantly moderated the relationship between taste and susceptibility $(\beta=0.19, p<.01)$ such that taste better predicted susceptibility in the experimental condition $(B=18.89, p<.001)$ than in the control condition $(B=13.57, p<.001)$.

\section{4 | DISCUSSION}

In this study, we tested (a) the impact of popular characterizing flavors on susceptibility to cigarillo use, as well as (b) mediation and moderation of the relationship between cigarillo flavor descriptors and susceptibility. We found that characterizing flavors increased susceptibility to use, while also increasing appeal ratings (taste, smell, and enjoyment) and social acceptability ratings. Taste and harm perceptions, in turn, influenced susceptibility to use. As expectations concerning taste was the sole variable that was both influenced by flavor descriptors and influenced susceptibility, we discovered that the key mediator of the relationship between flavor descriptors and susceptibility to use is taste perception. Among young adult nonusers of tobacco, characterizing flavors in cigarillos increase the favorability of expectations concerning the taste of cigarillos, and greater expectations of taste increase susceptibility to use. We also found evidence of moderation by taste perception; characterizing flavors increased the impact of expectations of taste on susceptibility to use. In fact, among participants who report high taste expectation ratings, exposure to cigarillos with characterizing flavors results in an 8-point increase (on a 100-point scale) in predicted susceptibility compared to those exposed to tobaccoflavored cigarillos. While other explicit perceptions-smell, enjoyment, social acceptability, and harm-either (a) are impacted by condition or (b) influence susceptibility, none of these perceptions did both. Interestingly, approach bias was neither impacted by flavor descriptors nor influenced susceptibility to cigarillo use, contrary to our hypothesis.

So, why is taste such a crucial factor when examining the relationship between cigarillo flavor descriptors and susceptibility to 
use? One explanation is that characterizing flavors mask the flavor and harshness of tobacco, thus, making it more appealing (U.S. Department of Health and Human Services, 2012). In fact, internal industry documentation indicates that reducing tobacco taste and harshness has been historically important to tobacco companies (Cummings, Morley, Horan, Steger, \& Leavell, 2002; Wayne \& Connolly, 2002). There is also recent qualitative research with smokers that supports the notion that flavoring hides tobacco's natural taste and makes smoking less harsh and more enjoyable (Sterling et al., 2015). When asked about flavored little cigars and cigarillos, a smoker said, "[flavor] adds to it as opposed to that same bland old tobacco taste. Cigarillo and cigars the tobacco is very harsh on my throat sometimes. But, for some reason flavors just make it a little bit more bearable." (p. 48). In addition to reducing harshness, taste cues may also elicit affective responses, as one smoker stated, "...yellow like the mango ones make me feel like" "okay this is going to be a good smoke" ... "Mango is like a day in the park." (p. 48). It also seems that taste and flavor are very closely related in smokers" minds. One cigarillo smoker said, "Cigarette smokers, they're smoking them because they want the nicotine. Whereas if you go out and buy like a Black \& Mild [a cigarillo brand] you usually look at the taste. That's what you look at is the flavor" (Sterling et al., 2015, p. 48). The reduction in harshness, elicitation of affect, and close mental association between flavored tobacco and taste perceptions may explain our findings that showed that taste is an important mechanism by which characterizing flavors increase susceptibility to cigarillo use.

We had anticipated that our implicit measure would result in novel findings regarding the role of approach bias in increasing susceptibility to cigarillo use; however, this was not the case. This might be due to the brief nature of exposure to the cigarillo packs in our study design. Interestingly, however, brief exposures to magazine advertisements for e-cigarette products among nonsmokers have increased implicit bias toward e-cigarettes in past research (Pokhrel et al., 2016). Both our study and the Pokhrel et al. (2016) study used brief exposures for nonusers. Therefore, it is possible that the reasons our study did not find such an effect are because (a) exposure to e-cigarettes has different effects on implicit bias than exposure to cigarillos, and perhaps because (b) we did not present our participants with ads (but rather cigarillo packs with flavor descriptions). It is possible that nonsmokers have a richer store of associations for e-cigarettes than cigarillos because advertising for e-cigarettes is less restricted than advertising for cigarillos, thus, increasing nonsmokers' exposure to e-cigarettes. It is also possible that because advertisements are meant to elicit implicit reactions, our presentation of more basic stimuli rather than cigarillo advertisements was not powerful enough to alter implicit bias. Thus, future research in this area may benefit from testing approach bias among nonusers with multiple (or longer) exposures to cigarillo products in advertisements (e.g., magazine ads) or to cigarillo stimuli with attractive design features.

In line with our hypotheses, perceptions of taste, smell, and enjoyment all increased due to characterizing flavors; these results replicate previous findings demonstrating that characterizing flavor descriptors increase appeal ratings (Meernik et al., 2018). We also found support for the prediction that characterizing flavors increase social acceptability as was observed in focus group research (Camenga et al., 2018; Wagoner et al., 2016). A key innovation of the present work was to model the impact of cigarillo flavor descriptors through implicit and explicit perceptions to susceptibility to use, and to test whether characterizing flavors change product perceptions and/or alter the weight attached to different product perceptions in predicting susceptibility to use. As far as we are aware, implicit reactions to flavor descriptors in tobacco have never been previously examined. Although cigarillos with characterizing flavors increased perceptions of taste, smell, enjoyment, and social acceptability and did not affect perceived harm, only one of these perceptions mattered for susceptibility to use-taste. Perceived taste mediated the effect of flavor descriptors on susceptibility and taste was the only significant predictor of susceptibility in the characterizing flavors condition. Descriptors of characterizing flavors also increased the impact of taste perceptions on susceptibility to cigarillo use.

These findings suggest that for young adult nonusers of tobacco, health warnings that only target the harms of cigarillo use will have less impact than warnings that simultaneously address expectations about the taste of cigarillos. This research has implications for public health and public policy, especially because approximately $73 \%$ of young adult users report using tobacco products with characterizing flavors (Villanti et al., 2017) and first use of a flavored tobacco product with characterizing flavor is positively related to subsequent tobacco use (as compared with first use of a tobacco-flavored product; Villanti et al., 2019). When considering new warnings for cigars and cigarillos with characterizing flavors, policy makers might consider targeting young adults' expectations regarding the taste of the products. Alternatively, due to the high rate of flavored tobacco use among young adults, paired with the increased susceptibility of tobacco nonusers when exposed to characterizing flavors (as demonstrated by this study), policy makers should consider limiting or banning characterizing flavors in cigars and cigarillos.

We recognize there are limitations to this first exploratory study. First, the study was conducted online rather than in the field or the lab. Prior research indicates that online convenience samples on Amazon Mechanical Turk provide valid and generalizable experimental findings that are equivalent to those obtained with national probability samples (Jeong et al., 2018). Second, we used a modified version of a validated self-report index rather than an objective measure of susceptibility and did not measure cigarillo smoking behavior. Measuring cigarillo use was not feasible in the present study. Future research would benefit from deploying longitudinal, experimental designs in order to both determine the durability of the impact of cigarillo flavor descriptors on product perceptions and to assess cigarillo use behavior-in addition to susceptibility to use-in the wake of exposure to cigarillo products with characterizing flavors. Finally, we acknowledge that present study's sample size was small ( $N=102$ ), though we note that we screened a very large number of potential participants online (2,276 individuals took our screener). Further studies with larger samples are warranted to corroborate the present findings. 
Notwithstanding these limitations, our findings indicate that characterizing flavors increase susceptibility to cigarillo use among nonsmokers through anticipated taste perceptions. Other perceptions (such as harm, social acceptability, etc.) do not fully explain the relationship between flavor descriptors and susceptibility. These results have potentially important implications for tobacco regulation and policy making in relation to banning or limiting characterizing flavors in little cigars and cigarillos.

\section{DISCLOSURE}

Research reported in this publication was supported by grant number P50CA180907 from the National Cancer Institute and the FDA Center for Tobacco Products (CTP). The content is solely the responsibility of the authors and does not necessarily represent the official views of the National Institutes of Health (NIH) or the Food and Drug Administration. This work was supported by a University of North Carolina at Chapel Hill Lineberger Comprehensive Cancer Center Developmental Award funded through the University Cancer Research Fund to Adam Goldstein. The Center for Regulatory Research on Tobacco Communication (CRRTC) also supported this work.

\section{CONFLICTS OF INTEREST}

The authors declare no conflicts of interest.

\section{ORCID}

Aya Avishai (iD https://orcid.org/0000-0002-0068-8883 Clare Meernik iD https://orcid.org/0000-0002-8564-1266 Allison J. Lazard iD https://orcid.org/0000-0002-2502-2850

\section{REFERENCES}

Byron, M. J., Lazard, A. J., Peters, E., Vu, H., Schmidt, A., \& Brewer, N. T. (2018). Effective formats for communicating risks from cigarette smoke chemicals. Tobacco Regulatory Science, 4(2), 16-29. https://doi. org/10.18001/TRS.4.2.2

Camenga, D. R., Fiellin, L. E., Pendergrass, T., Miller, E., Pentz, M. A., \& Hieftje, K. (2018). Adolescents' perceptions of flavored tobacco products, including E-cigarettes: A qualitative study to inform FDA tobacco education efforts through videogames. Addictive Behaviors, 82, 189-194. https://doi.org/10.1016/j.addbeh.2018.03.021

Cummings, K. M., Morley, C. P., Horan, J. K., Steger, C., \& Leavell, N. R. (2002). Marketing to America's youth: Evidence from corporate documents. Tobacco Control, 11(suppl 1), i5-i17. https://doi.org/10.1136/ tc.11.suppl_1.i5

DiFranza, J. R., Wellman, R. J., Sargent, J. D., Weitzman, M., Hipple, B. J., \& Winickoff, J. P. (2006). Tobacco promotion and the initiation of tobacco use: Assessing the evidence for causality. Pediatrics, 117(6), e1237-e1248. https://doi.org/10.1542/peds.2005-1817

Feirman, S. P., Lock, D., Cohen, J. E., Holtgrave, D. R., \& Li, T. (2015). Flavored tobacco products in the United States: A systematic review assessing use and attitudes. Nicotine \& Tobacco Research, 18(5), 739749. https://doi.org/10.1093/ntr/ntv176

Greenwald, A. G., McGhee, D. E., \& Schwartz, J. L. (1998). Measuring individual differences in implicit cognition: The implicit association test. Journal of Personality and Social Psychology, 74(6), 1464. https:// doi.org/10.1037/0022-3514.74.6.1464

Gritz, E., Prokhorov, A., Suchanek Hudmon, K., Mullin Jones, M., Rosenblum, C., Chang, C.-C., ... de Moor, C. (2003). Predictors of susceptibility to smoking and ever smoking: A longitudinal study in a triethnic sample of adolescents. Nicotine \& Tobacco Research, 5(4), 493-506. https://doi.org/10.1080/1462220031000118568

Harrell, M. B., Loukas, A., Jackson, C. D., Marti, C. N., \& Perry, C. L. (2017). Flavored tobacco product use among youth and young adults: What if flavors didn't exist? Tobacco Regulatory Science, 3(2), 168-173. https://doi.org/10.18001/TRS.3.2.4

Henriksen, L., Feighery, E. C., Wang, Y., \& Fortmann, S. P. (2004). Association of retail tobacco marketing with adolescent smoking. American Journal of Public Health, 94(12), 2081-2083. https://doi. org/10.2105/AJPH.94.12.2081

Huang, L. L., Baker, H. M., Meernik, C., Ranney, L. M., Richardson, A., \& Goldstein, A. O. (2017). Impact of non-menthol flavours in tobacco products on perceptions and use among youth, young adults and adults: A systematic review. Tobacco Control, 26(6), 709-719.

Jeong, M., Zhang, D., Morgan, J. C., Ross, J. C., Osman, A., Boynton, M. H., ... Brewer, N. T. (2018). Similarities and differences in tobacco control research findings from convenience and probability samples. Annals of Behavioral Medicine, 53(5), 476-485. https://doi. org/10.1093/abm/kay059

Karpinski, A., \& Steinman, R. B. (2006). The single category implicit association test as a measure of implicit social cognition. Journal of Personality and Social Psychology, 91(1), 16. https://doi. org/10.1037/0022-3514.91.1.16

Kowitt, S., Meernik, C., Baker, H., Osman, A., Huang, L. L., \& Goldstein, A. O. (2017). Perceptions and experiences with flavored non-menthol tobacco products: A systematic review of qualitative studies. International Journal of Environmental Research and Public Health, 14(4), 338. https://doi.org/10.3390/ijerph14040338

Lazard, A. J., Byron, M. J., Vu, H., Peters, E., Schmidt, A., \& Brewer, N. T. (2019). Website designs for communicating about chemicals in cigarette smoke. Health Communication, 34(3), 333-342.

Meernik, C., Ranney, L. M., Lazard, A. J., Kim, K. S., Queen, T. L., Avishai, A., ... Goldstein, A. O. (2018). The effect of cigarillo packaging elements on young adult perceptions of product flavor, taste, smell, and appeal. PLoS ONE, 13(4), e0196236. https://doi.org/10.1371/journ al.pone.0196236

Ostafin, B. D., \& Palfai, T. P. (2006). Compelled to consume: The Implicit Association Test and automatic alcohol motivation. Psychology of Addictive Behaviors, 20(3), 322. https://doi.org/10.1037/0893-164X.20.3.322

Palfai, T. P., \& Ostafin, B. D. (2003). Alcohol-related motivational tendencies in hazardous drinkers: Assessing implicit response tendencies using the modified-IAT. Behaviour Research and Therapy, 41(10), 1149-1162.

Pepper, J. K., Emery, S. L., Ribisl, K. M., Southwell, B. G., \& Brewer, N. T. (2014). Effects of advertisements on smokers' interest in trying e-cigarettes: The roles of product comparison and visual cues. Tobacco Control, 23(suppl 3), iii31-iii36. https://doi.org/10.1136/tobaccocon trol-2014-051718

Pierce, J. P., Choi, W. S., Gilpin, E. A., Farkas, A. J., \& Merritt, R. K. (1996). Validation of susceptibility as a predictor of which adolescents take up smoking in the United States. Health Psychology, 15(5), 355. https://doi.org/10.1037/0278-6133.15.5.355

Pokhrel, P., Fagan, P., Herzog, T. A., Chen, Q., Muranaka, N., Kehl, L., \& Unger, J. B. (2016). E-cigarette advertising exposure and implicit attitudes among young adult non-smokers. Drug and Alcohol Dependence, 163, 134-140. https://doi.org/10.1016/j.druga Icdep.2016.04.008

Sterling, K. L., Fryer, C. S., Nix, M., \& Fagan, P. (2015). Appeal and impact of characterizing flavors on young adult small cigar use. Tobacco Regulatory Science, 1(1), 42-53. https://doi.org/10.18001/ TRS.1.1.5

Swedish Match North America. (2015). Tobacco: Cigars 2015. Retrieved March 15, 2016, from http://www.cspnet.com/print/csp-magazine/ article/tobacco-cigars-2015? page $=0 \% 2 \mathrm{C} 2$. 
US Department of Health and Human Services. (2012). Preventing tobacco use among youth and young adults: A report of the Surgeon General. Atlanta, Georgia.

Van De Ven, M. O., Engels, R. C., Otten, R., \& Van Den Eijnden, R. J. (2007). A longitudinal test of the theory of planned behavior predicting smoking onset among asthmatic and non-asthmatic adolescents. Journal of Behavioral Medicine, 30(5), 435-445. https://doi. org/10.1007/s10865-007-9119-2

Villanti, A. C., Johnson, A. L., Ambrose, B. K., Cummings, K. M., Stanton, C. A., Rose, S. W., ... Hyland, A. (2017). Flavored tobacco product use in youth and adults: Findings from the first wave of the PATH study (2013-2014). American Journal of Preventive Medicine, 53(2), 139-151. https://doi.org/10.1016/j.amepre.2017.01.026

Villanti, A. C., Johnson, A. L., Glasser, A. M., Rose, S. W., Ambrose, B. K. Conway, K. P., ... Hyland, A. (2019). Association of flavored tobacco use with tobacco initiation and subsequent use among US youth and adults, 2013-2015. JAMA Network Open, 2(10), e1913804. https:// doi.org/10.1001/jamanetworkopen.2019.13804

Wagoner, K. G., Cornacchione, J., Wiseman, K. D., Teal, R., Moracco, K. E., \& Sutfin, E. L. (2016). E-cigarettes, hookah pens and vapes: Adolescent and young adult perceptions of electronic nicotine delivery systems. Nicotine \& Tobacco Research, 18(10), 2006-2012.

Wakefield, M., Germain, D., Durkin, S., Hammond, D., Goldberg, M., \& Borland, R. (2012). Do larger pictorial health warnings diminish the need for plain packaging of cigarettes? Addiction, 107(6), 1159-1167. https://doi.org/10.1111/j.1360-0443.2012.03774.x
Wang, T. W., Asman, K., Gentzke, A. S., Cullen, K. A., Holder-Hayes, E., Reyes-Guzman, C., ... King, B. A. (2018). Tobacco product use among adults - United States, 2017. Morbidity and Mortality Weekly Report, 67(44), 1225-1232. https://doi.org/10.15585/mmwr. $\mathrm{mm} 6744 \mathrm{a} 2$

Wayne, G. F., \& Connolly, G. N. (2002). How cigarette design can affect youth initiation into smoking: Camel cigarettes 1983-93. Tobacco Control, 11(suppl 1), i32-i39. https://doi.org/10.1136/tc.11.suppl_1. i32

\section{SUPPORTING INFORMATION}

Additional Supporting Information may be found online in the Supporting Information section.

How to cite this article: Avishai A, Meernik C, Goldstein AO, Lazard AJ, Ranney LM, Sheeran P. Impact and mechanisms of cigarillo flavor descriptors on susceptibility to use among young adult nonusers of tobacco. J Appl Soc Psychol.

2020;50:699-708. https://doi.org/10.1111/jasp.12706 